\title{
ESTRATÉGIA DE DIGITALIZAÇÃO DO PARQUE IMOBILIÁRIO DA EDP GLOBAL SOLUTIONS
}

\author{
André Monteiro ${ }^{(1)}$, Gustavo Leal $^{(2)}$, João Hormigo ${ }^{(3)}$ \\ (1) bimTEC, Porto \\ (2) bimTEC, Porto \\ (3) EDP Global Solutions, Lisboa
}

\begin{abstract}
Resumo
A digitalização de processos é uma diretiva cada vez mais presente e transversal nas empresas de grande dimensão. No caso do Grupo EDP, esta estratégia estende-se à empresa do grupo responsável pela gestão do parque imobiliário, a EDP Global Solutions, que encontrou na metodologia BIM a melhor forma de acelerar o processo de digitalização, através da criação de modelos de informação (BIM) em que seja possível intersetar os benefícios da utilização de interfaces gráficas tridimensionais para centralização e consulta paramétrica da informação de projeto, obra e exploração dos ativos de modo a desbloquear um conjunto de aplicações de processamento de meta-dados para otimização da gestão interna dos ativos imobiliários.

O presente artigo descreve a estratégia de digitalização do parque imobiliário da EDP Global Solutions, atualmente em desenvolvimento com o apoio da empresa consultora bimTEC segundo os três vetores operacionais seguintes: 1) preparação de equipa interna para gerir processos BIM envolvendo as empresas prestadoras de serviços; 2) criação dos ativos digitais do parque imobiliário da empresa através da compilação, centralização e modelação BIM da informação dos edifícios; 3) preparação estratégica da infraestrutura necessária para obtenção do gémeo digital dos edifícios.
\end{abstract}

\section{Introdução}

A integração e aplicação de processos BIM na EDP Global Solutions (EDPGS), empresa responsável pela gestão do parque imobiliário do Grupo EDP, foi identificada internamente como estratégica para a gestão de projetos e a gestão da manutenção dos seus ativos imobiliários. Recorrendo a uma empresa consultora, a bimTEC, foi desenvolvida uma estratégia idealizada com base nos dois macro objetivos seguintes: 1) apoiar os processos de gestão de projetos, obras e manutenção de edifícios; 2) digitalizar os ativos imobiliários da empresa de modo a potenciar a utilização de aplicações relacionadas com a gestão e manutenção 
de ativos enquadradas nas tecnologias emergentes de realidade virtual, realidade aumentada, internet das coisas , "big data" e algoritmos preditivos.

Com início em 2018, as primeiras fases do processo focaram as tradicionais componentes de adoção de uma metodologia BIM [1], incluindo a definição de uma equipa de trabalho, a criação de uma infraestrutura interna de trabalho suportado em ferramentas BIM, a formação da equipa de trabalho e o teste de processos num projeto-piloto. Sendo a equipa da EDPGS essencialmente focada nos processos de gestão de projetos, obras e manutenção de ativos, a estratégia de formação e aquisição de "competências BIM" focou-se bastante na utilização de plataformas colaborativas BIM e na preparação da equipa para definir requisitos de modelação e coordenação de modelos em moldes semelhantes aos propostos no modelo inglês da PAS:1192 [2], entretanto adaptado para a norma internacional ISO 19650 [3].

O arranque da empreitada de construção da Sede EDP II em Lisboa foi outro fator importante para a decisão de iniciar o processo de integração de processos BIM na EDPGS, tratando-se de uma boa oportunidade para colocar em prática as metodologias de modelação, coordenação e colaboração suportadas em ferramentas BIM. Encontrando-se atualmente em desenvolvimento, o intuito do processo passa por reduzir algum do risco associado à empreitada através da otimização de processos de coordenação e comunicação, e obter uma tela final do edifício em formato BIM, configurada de acordo com os requisitos específicos da utilização de modelos na fase de exploração do ativo imobiliário.

A modelação do parque imobiliário da EDPGS assenta na estratégia global do Grupo EDP para digitalização e sistematização de processos, centralização de dados e aplicação de tecnologias inovadoras. No caso particular dos ativos imobiliários, o processo de digitalização começa na criação de um modelo BIM representativo do edifício que possa ser utilizado para navegação espacial e consulta da informação atualizada sobre a exploração dos edifícios e sobre a gestão e manutenção dos espaços e equipamentos. O processo de digitalização dos ativos encontra-se atualmente em desenvolvimento, com o foco inicial a incidir sobre três edifícios estratégicos, nomeadamente, a sede em Lisboa e os edifícios de escritórios e serviços no Porto e em Coimbra. Do ponto de vista conceptual, a estratégia de digitalização do parque imobiliário da EDPGS contempla um cenário em que a empresa prestadora de serviços de gestão e manutenção dos ativos da EDP adote um workflow que permita a constante atualização do modelo BIM do ativo imobiliário, por forma a que a equipa da EDPGS tenha acesso à informação de manutenção e exploração dos ativos numa plataforma onde a informação se encontre centralizada, enquadrada num modelo BIM e navegável a partir de um computador ou dispositivo móvel.

Do ponto de vista operacional, a modelação BIM do parque imobiliário da EDPGS consiste na compilação, organização, processamento e centralização dos dados dos ativos, processos necessários para preparar a aplicação de tecnologias avançadas como a realidade aumentada, a inteligência artificial e os sistemas preditivos.

\section{Implementação BIM na EDP Global Solutions}

\subsection{BIM para Gestores}

O grupo de trabalho constituído no âmbito do processo de implementação da metodologia BIM na EDPGS integra colaboradores que na sua maioria desempenham atividades de gestão de equipas e/ou fornecedores, normalmente externos à empresa, dividindo-se nas três áreas funcionais seguintes: 
- Projetos (3 colaboradores)

- Espaços (4 colaboradores)

- Hard Services (4 colaboradores)

Sendo o âmbito de atuação do grupo algo heterogéneo, foram estabelecidos objetivos e atividades transversais a todo o grupo, mas também metas e processos específicos por área funcional, conforme esquematizado na Figura 1. A definição de requisitos BIM para contratação de equipas, a avaliação de processos de modelação e organização de formatos de entrega de modelos, a utilização de plataformas colaborativas e a consulta de informação em modelos BIM são competências indispensáveis para um gestor que pretenda trabalhar com equipas que utilizem ativamente uma metodologia BIM no seu workflow diário, fazendo deste modo parte do programa formativo transversal a todo o grupo. $\mathrm{Na}$ prática, este tipo de competências vem a traduzir-se na capacidade de produzir um documento formal de requisitos de informação BIM, habitualmente designado "Caderno de Encargos BIM" ou "Employer's Information Requirements" (EIR), de avaliar uma proposta de programa e metodologia de trabalhos BIM, habitualmente designado "Plano de Execução BIM" ou "BIM Execution Plan" (BEP), de consultar e gerir documentos (incluindo modelos) numa plataforma colaborativa, e de verificar o cumprimento ao longo do projeto das especificações definidas nos referidos documentos.

\section{Implementação da metodologia BIM na EDP Global Solutions}

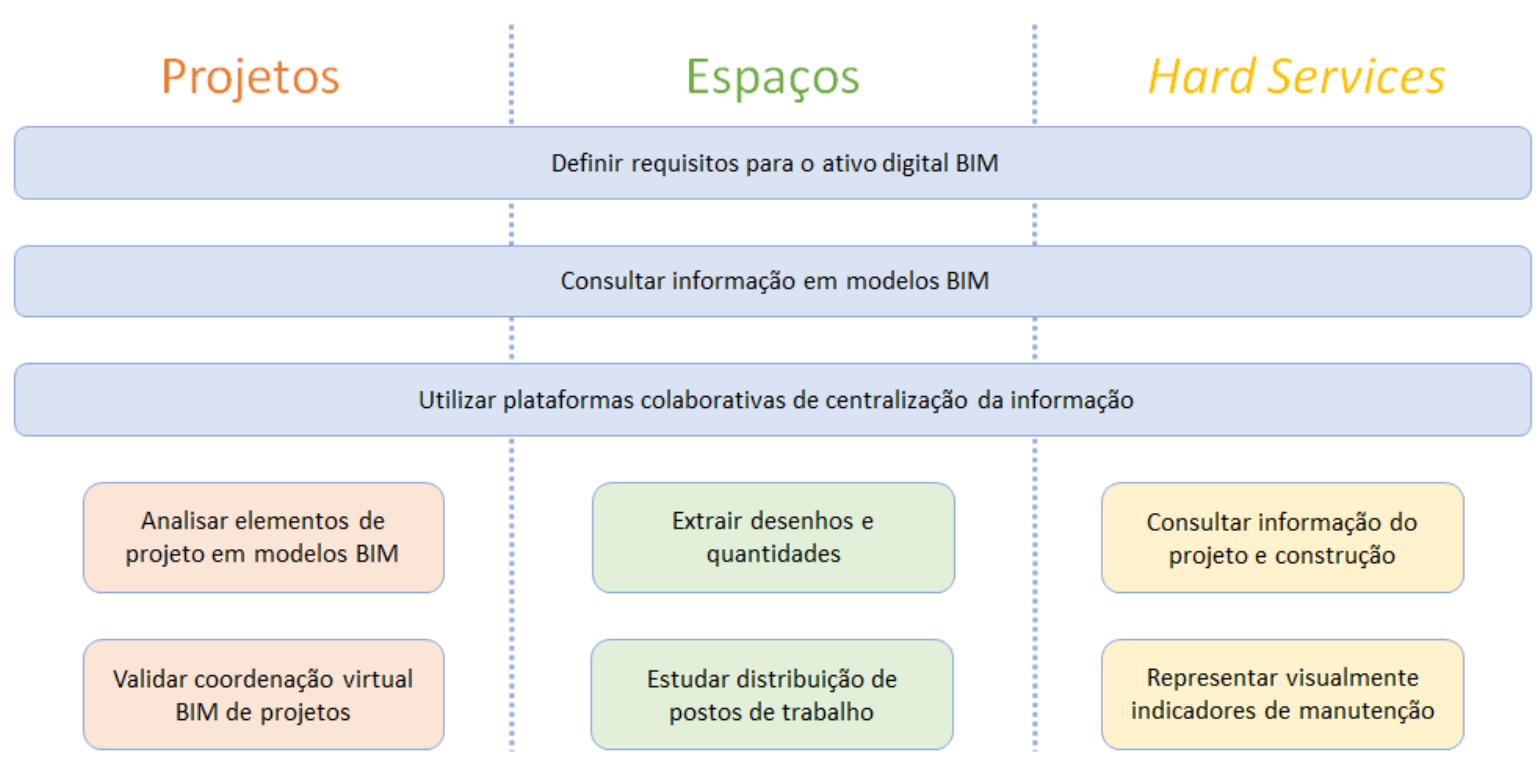

Figura 1: Objetivos da implementação da metodologia BIM na EDP Global Solutions.

\subsection{BIM para (Gestores de) Projeto}

As competências transversais para qualquer tipo de gestor que pretenda trabalhar segundo uma metodologia BIM serão à partida suficientes para um gestor de projeto que pretenda acompanhar o desenvolvimento dos trabalhos das equipas encarregues de produzir os modelos e utilizar as ferramentas. No entanto, para exercer um papel mais ativo do ponto de vista da gestão e coordenação de projeto e para tirar maior partido das ferramentas que terá ao seu 
dispor, o gestor de projeto beneficiará com o desenvolvimento de competências na coordenação de modelos BIM e na inspeção dinâmica de modelos. Neste sentido, o programa formativo da equipa de (gestão de) Projeto da EDPGS focou bastante a componente de coordenação via Clash Detection (configuração e aplicação de rotinas de deteção automática de colisões) e a componente de extração de tabelas de quantidades e informação a partir de modelos.

Sendo o gestor de projeto figura presente ao longo do ciclo de vida da construção do edifício, também na fase de execução da empreitada existem aplicações da metodologia BIM que contribuem para apoiar no processo de análise e decisão do gestor. Neste âmbito, as atividades formativas incidiram sobre a utilização de ferramentas de ligação de modelos a planeamentos de obra (4D BIM) e sobre a utilização de plataformas colaborativas com mecanismos de gestão documental que permitem a integração de pedidos de esclarecimento diretamente a partir dos modelos e desenhos.

\subsection{BIM para (Gestores de) Espaços}

Das três áreas funcionais da EDPGS, a área de (gestão de) Espaços é a que mais se aproxima de uma tradicional equipa projetista de arquitetura. A equipa é responsável pela definição de interiores dos imóveis, pela remodelação dos espaços e pelo cadastro e gestão das áreas de trabalho atribuídas a cada empresa do Grupo EDP de acordo com as dinâmicas internas de mobilidade dos postos de trabalho.

O trabalho de desenho verifica-se sobretudo na componente de remodelação, com as ferramentas BIM a substituírem as tradicionais ferramentas CAD como ambiente de modelação para produção de desenhos de interiores. Um requisito fundamental à implementação deste tipo de procedimento é a pré-existência do modelo do edifício, sendo um dos motivos que justifica a digitalização do parque imobiliário da empresa e a preparação das equipas de gestão de projeto para exigirem telas finais em formato BIM.

A outra componente significativa da atividade da equipa de Espaços prende-se com a gestão de postos de trabalho das empresas do Grupo EDP. O que tradicionalmente é feito com recurso a uma base de dados ou folha de cálculo acompanhada de um desenho ilustrativo, passa, através da utilização de um modelo BIM, a ser algo totalmente integrado, com o modelo a funcionar como base de dados e ambiente de visualização. A conceção de um sistema de gestão de espaços a partir de um modelo BIM obrigou à criação de um workflow que sistematiza a introdução e extração dinâmica da informação dos modelos e habilita a identificação visual dos espaços e postos de trabalhos por critério (utilizado/não utilizado, e empresa do Grupo EDP).

De acordo com o workflow, a introdução de informação poderá ser realizada via base de dados ou via modelo. Independentemente do meio escolhido para a entrada de informação, a base de dados global será sempre atualizada de modo a obter uma fonte única e central de informação.

\subsection{BIM para (Gestores de) Hard Services}

A equipa dos Hard Services é responsável pela supervisão dos prestadores de serviços de gestão e manutenção dos edifícios, com quem são articuladas as operações relacionadas com as intervenções nos edifícios e a encomenda de equipamentos e materiais.

A digitalização de processos através da implementação de ferramentas e processos BIM tende a ser mais acessível na fase de projeto e construção, dada a proliferação de ferramentas e metodologias especificamente orientadas para essas fases. Na fase de exploração o cenário é menos favorável. Existem menos ferramentas específicas de BIM-FM, isto é, ferramentas que integrem modelos BIM com processos de manutenção de edifícios; existem menos aplicações 
documentadas da aplicação de uma metodologia BIM integrada com a gestão e manutenção de ativos; sobretudo, raramente existem os ativos digitais, leia-se, uma base de dados (ou modelo BIM) para a implementação de uma metodologia dessa natureza [4] [5]. Com efeito, o último ponto assume especial importância, visto que a desagregação da informação entre desenhos de projeto, desenhos de telas finais, cadastro de equipamentos e ativos por parte do dono/utilizador do edifício, cadastro de equipamentos e ativos por parte do prestador de serviços de manutenção, cadastro de equipamentos na gestão técnica centralizada, entre outros, resulta na multiplicação da informação por diversos locais, normalmente de forma inconsistente e incompleta. A criação do ativo digital tem, portanto, o objetivo maior de consolidar toda esta informação num único local com vista à criação de condições para a implementação de funcionalidades especificamente relacionadas com a gestão e manutenção de ativos [6].

As atividades formativas para a equipa de Hard Services focaram sobretudo a troca de informação entre bases de dados, sobretudo, em formato de tabela compatível com folhas de cálculo e modelos BIM. Outro vetor explorado foi a consulta de informação de manutenção diretamente nos modelos, quer através das tabelas de informação diretamente integradas nos modelos, quer através de mapas 2D e 3D com a atribuição de códigos de cores em função do estado do equipamento/ativo.

\section{Aplicação na empreitada de construção do edifício Sede EDP II}

A empreitada de construção do edifício Sede EDP II em Lisboa foi um forte catalisador para a adoção de uma metodologia BIM estruturada que visa, por parte da entidade executante, a utilização de processos BIM durante a fase de construção e a produção de telas finais em formato BIM compatíveis com uma futura utilização na fase de exploração do ativo. A empreitada teve início em fevereiro de 2020 e tem uma duração prevista de dois anos.

\subsection{Fase de concurso e contratação}

$\mathrm{Na}$ fase de concurso da empreitada foi adotada uma metodologia de definição de requisitos de informação, apresentação de proposta de trabalho e formalização de metodologia BIM inspirada nas diretivas da norma inglesa PAS 1192. Esta opção foi justificada pela necessidade de definir de forma criteriosa os procedimentos relacionados com a aplicação da metodologia BIM que se pretendia ver aplicar pela entidade executante ao longo da fase de construção, com especial ênfase na produção das telas finais.

Tal como previsto na PAS 1192, o processo teve início com a criação do EIR (da fase de construção) onde foram definidos os requisitos de informação para a metodologia BIM a apresentar pelas entidades concorrentes. As especificações do EIR definiam dois tipos de requisitos fundamentais para a aplicação da metodologia BIM na fase de construção, nomeadamente, para a coordenação dos projetos e para a produção dos modelos de telas finais. $\mathrm{Na}$ etapa seguinte foram analisadas as propostas de BEP apresentadas pelas entidades adjudicatárias, sendo de destacar a variabilidade no formalismo das propostas apresentadas, com algumas entidades a dedicaram apenas algumas linhas da sua proposta à resposta aos requisitos BIM, enquanto outras apresentaram um documento formal respondendo diretamente aos requisitos de informação do EIR com uma metodologia de trabalho e a demonstração de recursos necessários à sua execução. A segunda abordagem constituiu naturalmente um fator de valorização por parte do dono de obra. Outro aspeto relevante foi o facto do BEP da entidade 
executante a quem foi adjudicada a obra prever um âmbito de aplicação da metodologia BIM mais alargado e ambicioso em relação ao que se encontrava previsto no EIR.

Para finalizar a etapa de contratação, foi criado, a partir do EIR do dono de obra e do BEP da entidade executante, o plano de gestão e publicação da informação, designado na PAS 1192 por "Master Information Delivery Plan" (MIDP). Neste documento, foi definida a metodologia BIM e o programa de trabalhos final para a fase de construção, vinculando as várias partes, em particular a entidade executante, às suas especificações. Tendo o documento sido produzido pela bimTEC na qualidade de consultor BIM, é de assinalar o envolvimento e contributo das restantes partes envolvidas, nomeadamente, dono de obra, fiscalização e entidade executante, que ao longo de diversas iterações contribuíram ativamente para otimização do documento de modo a obter-se uma versão final perfeitamente alinhada com a visão das diferentes partes para a operacionalização da metodologia BIM ao longo da empreitada de construção.

\subsection{Metodologia BIM e programa de trabalhos}

O MIDP da empreitada de construção da Sede EDP II contempla três grandes vetores de desenvolvimento, correspondentes às três principais fases da empreitada seguintes:

1. Coordenação dos projetos: Prevê a análise dos projetos, a modelação BIM sempre que necessário e a coordenação virtual através da inspeção visual dos modelos, da apreciação técnica dos projetos e da realização de clash detection para identificação de potenciais situações de coordenação a resolver. Os resultados desta fase incluem a entrega dos modelos de coordenação, de um relatório com a interpretação e apreciação crítica dos resultados da coordenação virtual, e de uma proposta de resolução de eventuais problemas;

2. Documentação de preparação: Contempla a criação do modelo de construção que resulta diretamente do modelo de coordenação da fase anterior, mas adaptado de acordo com as especificações da entidade executante. São ainda criados modelos de preparação de obra que derivam do modelo de construção, detalhados de acordo com os requisitos de produção da documentação de apoio aos trabalhos de construção, instalação e fabrico. Os resultados desta fase incluem a entrega de pacotes de elementos de preparação (modelos e desenhos) organizados por localização (piso e edifício);

3. Modelação as-built para FM: A criação do modelo as-built surge a partir da atualização periódica e sistemática do modelo de construção. Para além do desenvolvimento geométrico do modelo, os requisitos de informação são assegurados através da classificação dos elementos do modelo de acordo com um sistema de classificação universal, neste caso o Uniformat II, e através da integração da ficha técnica dos ativos. Os documentos das fichas técnicas são carregados e organizados numa pasta da plataforma colaborativa e o endereço eletrónico dos documentos introduzido nos respetivos elementos dos modelos.

Para além dos aspetos relacionados com as entregas formais, existe uma componente processual ligada à metodologia de comunicação e gestão documental bastante importante. Aproveitando a implementação de uma plataforma colaborativa para submissão dos ficheiros, foi incrementado o âmbito da sua utilização através da integração da gestão de pedidos de esclarecimento na plataforma em substituição do email. A adoção desta funcionalidade foi motivada pela vontade de melhor controlar o processo de troca deste tipo de informação e de circunscrever estas interações a uma metodologia organizada e formalmente balizada. 


\section{Digitalização dos ativos imobiliários}

\section{1. Âmbito}

A digitalização dos ativos imobiliários tem como objetivo obter um modelo tridimensional dos edifícios do parque imobiliário da empresa onde seja possível consultar, por um lado, as especificações técnicas dos ativos dos edifícios, e por outro, a informação de exploração dos ativos ligada às operações de utilização e manutenção de espaços e equipamentos. Encontrandose atualmente em desenvolvimento, o processo irá decorrer de forma faseada, começando pelos edifícios com maior índice de utilização, nomeadamente a sede em Lisboa e os edifícios de escritórios e serviços no Porto e em Coimbra. Na próxima fase pretende-se que o processo seja replicado nos restantes edifícios relevantes do ponto de vista da gestão e manutenção de ativos. $\mathrm{O}$ processo encontra-se esquematizado na Figura 2.

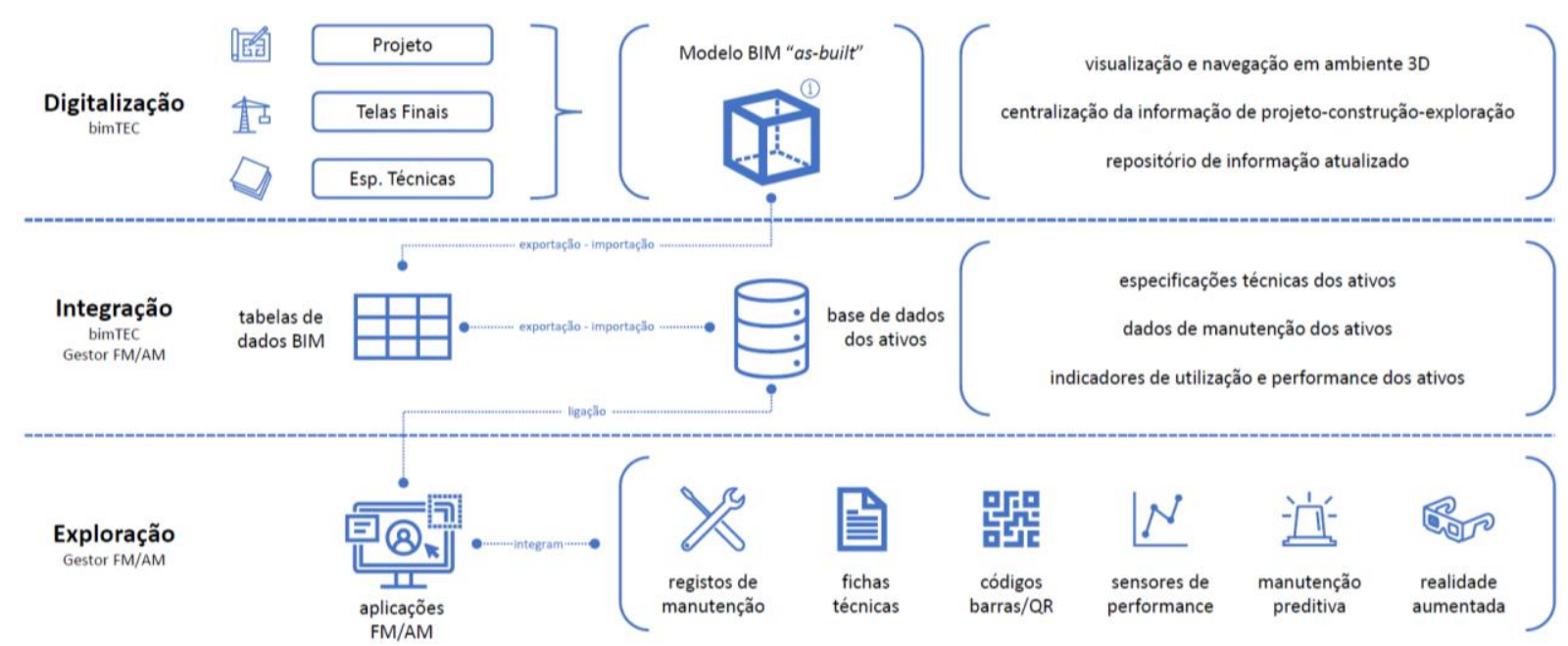

Figura 2: Estratégia de digitalização do parque imobiliário da EDP Global Solutions.

\subsection{Pré-requisitos do ativo digital}

No processo de digitalização do parque imobiliário da EDPGS foram identificados os prérequisitos seguintes:

- Bases de modelação: Para criação do ativo digital são necessárias, numa primeira fase, bases de modelação atualizadas. A forma mais precisa de desenvolver um modelo neste tipo de enquadramento passa pela utilização dos desenhos de telas finais como base de modelação, no entanto, a utilização das "tradicionais" telas finais apresenta vários problemas: 1) os desenhos poderão não ser uma representação exata do $a$ s-built; 2) é frequente as telas finais dos edifícios de escritórios não incluírem a arquitetura dos interiores; 3) a informação poderá encontrar-se desatualizada relativamente às intervenções realizadas no âmbito das operações de manutenção e/ou remodelação; 4) a identificação dos equipamentos nas telas finais poderá estar desfasada da notação utilizada pelo prestador de serviços de manutenção e gestão de ativos. Tendo em conta estes aspetos, a utilização de desenhos/modelos como bases de modelação deverá ser sempre complementada com um levantamento do existente, realizado presencialmente no local [7]; 
- Levantamento: Nas situações em que o edifício já se encontra construído, a realização de um levantamento das condições locais é um fator bastante importante para confirmar as disposições das bases de modelação. Caso não existam telas finais ou estas se encontrem demasiado desatualizadas, o levantamento poderá ser realizado essencialmente das duas formas seguintes: 1) levantamento manual através do registo fotográfico, medição e desenho manual das condições locais; 2) levantamento "laser scanning" para criação de nuvem de pontos a utilizar como referência na construção do modelo BIM;

- Modelação BIM e centralização da informação: A criação de um modelo BIM-FM/AM (FM-Facilities Management/AM-Asset Management), isto é, um modelo para manutenção e gestão de ativos, deverá ter em conta os princípios fundamentais seguintes: 1) nível de detalhe geométrico médio a baixo (LOD 300/200), suficiente para a identificação inequívoca do elemento enquadrado no espaço mas sem detalhe que sobrecarregue graficamente e prejudique a navegação e consulta do modelo; 2) classificação uniforme dos elementos segundo um sistema de classificação universal (como por exemplo, o Uniformat II ou o Uniclass) e identificação dos elementos conforme definido na base de dados de gestão e manutenção de ativos; 3) associação das especificações técnicas dos materiais e equipamentos, preferencialmente, a partir de uma ligação direta à respetiva ficha técnica;

- Ligação à base de dados FM/AM: Definição do protocolo de ligação à base de dados de gestão e manutenção de ativos, a realizar de uma das formas seguintes: 1) transferência de dados entre modelo BIM e base de dados através do cruzamento da informação por meio de uma tabela intermediária; 2) ligação indireta do modelo à base de dados através da criação de uma ligação que faz o pedido à base de dados sempre que seja necessário importar informação para o modelo; 3) ligação direta da base de dados ao modelo através do envio automático da informação da base de dados para o modelo sempre que for registada uma atualização da primeira. Das três possibilidades identificadas, a primeira constitui a solução mais abrangente e ideal para a conceptualização inicial do sistema a implementar; a segunda constitui a solução ideal, de modo a que a informação possa ser acedida no modelo sem criar um processo que sobrecarregue ambos os sistemas; a terceira tem interesse para plataformas de acompanhamento em tempo real e monitorização de espaços e equipamentos.

\subsection{Aplicações práticas e gémeo digital}

A mais prática e direta aplicação do ativo digital passa pela democratização do acesso à informação. Uma das principais dificuldades de uma empresa com vários ativos imobiliários que subcontrata a gestão e manutenção de ativos prende-se com a dificuldade em aceder à informação atualizada do edifício. A informação encontra-se dispersa por equipas diferentes, por empresas diferentes e por locais diferentes. A utilização de plataformas de gestão de ativos digitais permite integrar os diversos ativos espalhados pelo país (ou mesmo pelo mundo) numa única interface e cruzar os dados para obter análises mais informadas sobre a sua utilização. Torna-se deste modo bastante mais acessível aos colaboradores da empresa acederem à informação que necessitam, reduzindo o tempo necessário para aceder à informação e a dependência de terceiros.

A criação do ativo digital é o pré-requisito essencial para desbloquear um conjunto de aplicações a implementar na fase de exploração do edifício que podem ser separadas nas duas categorias seguintes: 
- Aplicações dependentes de interface gráfica tridimensional: correspondentes às aplicações que dependem da existência de um modelo 3D, incluindo: 1) disponibilização de um protótipo tridimensional do edifício aos colaboradores da empresa para consulta e navegação remota; 2) utilização de realidade virtual para navegação do edifício em primeira pessoa; 3 ) utilização de realidade aumentada para consulta ao vivo de informações dos equipamentos e visualização de instalações embebidas ou escondidas; 4) ligação de equipamentos a modelos BIM via implementação de códigos $\mathrm{QR}$, códigos de barras ou NFC; 5) integração da gestão técnica centralizada com central de sensorização e monitorização;

- Aplicações de processamento de meta dados: não dependendo de uma interface gráfica tridimensional mas beneficiando da centralização da informação processada num modelo BIM, correspondem a aplicações geralmente implementadas parcialmente mas que apenas poderão funcionar de forma ótima quando integradas entre si, incluindo: 1) desenvolvimento de algoritmos preditivos de auxílio à manutenção; 2) medição da performance energética do edifício e dos espaços; 3 ) registo de métricas de utilização dos ativos através da incorporação de sensores de monitorização; 4) aplicação de inteligência artificial para otimização de consumos energéticos com base em dados de utilização dos espaços.

Uma implementação transversal de várias destas aplicações com um modelo BIM permitem obter o gémeo digital do edifício, isto é, uma prototipagem digital do edifício atualizada em tempo real onde é possível consultar informação estática ou dinâmica sobre o edifício e os seus ativos [8] [9]. O conceito de gémeo digital está diretamente relacionado com o conceito da internet das coisas ou "Internet of Things" (IoT), que no caso da gestão e manutenção dos edifícios permitiria às equipas da EDPGS, por exemplo, consultar remotamente a temperatura de um espaço ou o número de postos de trabalho disponíveis em determinada sala a partir de um modelo BIM.

\section{Conclusões}

A implementação da metodologia BIM-FM/AM idealizada para a EDPGS pode ser resumida nos três vetores seguintes:

1. Digitalização: Transposição da informação do projeto e da construção para um contentor digital de informação através da criação do modelo BIM onde se encontra centralizada a informação do ativo. A digitalização visa centralizar a informação de utilização dos edifícios e beneficiar da utilização de uma interface gráfica para análise e exploração tridimensional dos ativos. Este processo desenvolve-se em duas vertentes: 1) preparação da equipa da EDPGS para obter ativos digitais em obras futuras; 2) modelação do parque imobiliário da EDPGS para cadastro digital dos ativos;

2. Integração: Ligação do modelo BIM às aplicações nativas de gestão e manutenção do ativo, através de processos expeditos para troca de informação entre bases de dados. A integração visa vincular informação de projeto e construção às aplicações de gestão da manutenção, e manter um repositório de informação atualizado com os dados de exploração. Os modelos BIM dos ativos imobiliários estão a ser preparados para trocar dados com a base de dados 
do prestador de serviços de gestão e manutenção. A criação da ligação dinâmica entre modelo BIM e base de dados do prestador de serviços de gestão e manutenção dependerá da plataforma escolhida para operacionalizar as aplicações de FM/AM;

3. Exploração: Exploração de aplicações potenciadas pelo processamento de dados de exploração do ativo e da sua integração com os ativos digitais suportados pelos modelos BIM. A exploração visa digitalizar operações de manutenção on site através da utilização de ferramentas de ligação visual dos ativos às bases de dados de FM/AM. Através da operacionalização do ativo digital com os dispositivos de sensorização, monitorização e realidade virtual, pretende-se obter um gémeo digital para consulta de indicadores de desempenho que se encontre ligado à gestão técnica centralizada e alimente os algoritmos de manutenção preditiva.

O sucesso desta última componente dependerá das aplicações e plataformas de BIM-FM/AM que ainda se encontram numa fase ascensional de maturação. Não obstante este facto, a preparação da infraestrutura BIM encerrará benefícios intermédios pela simples concretização da centralização e disponibilização da informação dos ativos por via de um ambiente gráfico tridimensional paramétrico.

\section{Referências}

[1] A. Monteiro, J. Lima, S. Henriques, L. Rodrigues e L. Ribeirinho, "Implementação BIM numa empresa de estudos e projetos de Engenharia (CENOR)," em $1^{o}$ Congresso Português de Building Information Modelling, Universidade do Minho, Guimarães, 2016.

[2] BSI, "PAS 1192-2:2013, Specification for information management for the capital/delivery phase of construction projects using building information modelling," BSI Standards Limited 2013, Reino Unido, 2013.

[3] ISO/TC 59/SC 13, "ISO/DIS 19650-1.2, Organization of information about construction works - Information management using building information modelling - Part 1: Concepts and principles," ISO, 2018.

[4] NBS, "10th Annual National BIM Report," NBS Enterprises Lts, Reino Unido, 2020.

[5] MT Højgaard, "Whitepaper - Efficient Digital Delivery of Construction with COBie," MT Højgaard, 2016.

[6] Vinci Facilities, "BIM for Facility Management - Handbook and technical specifications for the digital model," Groupe Moniteur, França, 2016.

[7] B. Bortoluzzi, I. Efremov, C. Medina, D. Sobieraj e J. McArthur, "Automating the creation of building information models for existing buildings," Automation in Construction, vol. 105, no 10.1016, p. 102838, 2019.

[8] S. Evans, C. Savian, A. Burns e C. Cooper, "Digital twins for the built environment, An introduction to the opportunities, benefits, challenges and risks," The Institution of Enginnering and Technology (IET), Atkins, Reino Unido, 2019.

[9] S. H. Khajavi, N. H. Motlagh, A. Jaribion, L. C. Werner e J. Holmström, "Digital Twin: Vision, Benefits, Boundaries, and Creation for Buildings," IEEE Access, vol. 7, 2019. 